\title{
Invasion and the evolution of speed in toads
}

\section{Cane toads seem to have honed their dispersal ability to devastating effect over the generations.}

Cane toads (Bufo marinus) are large anurans (weighing up to $2 \mathrm{~kg}$ ) that were introduced to Australia 70 years ago to control insect pests in sugar-cane fields. But the result has been disastrous because the toads are toxic and highly invasive. Here we show that the annual rate of progress of the toad invasion front has increased about fivefold since the toads first arrived; we find that toads with longer legs can not only move faster and are the first to arrive in new areas, but also that those at the front have longer legs than toads in older (long-established) populations. The disaster looks set to turn into an ecological nightmare because of the negative effects invasive species can have on native ecosystem $\mathrm{s}^{1,2}$; over many generations, rates of invasion will be accelerated owing to rapid adaptive change in the invader ${ }^{3}$, with continual 'spatial selection' at the expanding front favouring traits that increase the toads' dispersal ${ }^{45}$.

Introduced to Queensland in 1935, cane toads have since expanded their range to encompass more than a million square kilometres of tropical and subtropical Australia ${ }^{6}$. We have radio-tracked toads (for methods, see supplementary information) at the invasion front $60 \mathrm{~km}$ east of Darwin and confirmed the astonishing locomotor performance in these animals, which move up to $1.8 \mathrm{~km}$ per night during the rainy months - far further than previously studied anurans? ${ }^{7}$. Does this remarkable ability result from selection for enhanced dispersal during the toads' Australian colonization history?

The morphological trait most often linked to locomotor ability in anurans is leg length, both among and within species. ${ }^{8}$. Our trials (see supplementary information) confirm that cane toads with relatively long legs are indeed faster over a short distance (regressing time taken to cover $1 \mathrm{~m}$ against residual leg length: $r=-0.44, n=29, P<0.02)$. But, more important, longer-legged toads moved further over $24 \mathrm{~h}$ (maximum displacement of radio-tracked toads versus relative leg length: $r=0.46, n$ $=21, P<0.04)$ and over three days $(r=0.58$, $n=21, P<0.006$; Fig. 1a). Longer legs therefore facilitate more rapid dispersal.

If the invasion process has been assisted by the evolution of improved dispersal ability among toads at the front, three consequences would be expected. First, longer-legged toads should be disproportionately common among the first wave of arrivals at any site. As the toad invasion front passed our study site, we measured relative leg lengths of all toads encountered over a 10-month period. Longer-legged toads were the first to pass through, followed by shorter-legged conspecifics (order of arrival versus relative leg length: $r=-0.34, n=552$, $P<0.0001$; Fig. 1b). Longer-legged toads therefore moved faster through the landscape. Second, toads at the invasion front should be longer-legged than toads from older populations. As predicted, longer-term historical analysis within Queensland populations shows that relative leg length is greatest in new arrivals and then declines over a 60 -year period (Fig. 1c; $r=-0.23, n=139, P<0.008$ ).

Third, the rate of progress of the toad invasion front should increase through time. As predicted, rates of frontal progress have con- sistently increased (Fig. 1d; time versus annual rate of spread, Pearson's $r=0.96, P<0.005)$. Toads expanded their range by about $10 \mathrm{~km}$ a year during the $1940 \mathrm{~s}$ to 1960 s, but are now invading new areas at a rate of over $50 \mathrm{~km}$ a year. Accordingly, previous predictions about the time course of future expansion of the toads' range' seriously underestimate their actual rates of movement.

These rapid shifts in toad morphology, locomotor speed and invasion velocity indicate that conservation biologists and managers need to consider the possibility of rapid adaptive change in invading organisms. If there is no fitness disadvantage to individual organisms at the invasion front, evolutionary forces are likely to fine-tune organismal traits in ways that facilitate more rapid expansion of the invading population ${ }^{10}$. Hence, control efforts against feral organisms should be launched as soon as possible, before the invader has had time to evolve into a more dangerous adversary. Benjamin L. Phillips, Gregory P. Brown, Jonathan K. Webb, Richard Shine School of Biological Sciences A08, University of Sydney, New South Wales 2006, Australia e-mail rics@bio.usyd.edu.au

1. Crossland, M.R. Ecography 23, 283-290 (2000).

2. Smith K G Biol. Conserv. 123,433-441(2005)

3. Cox G.W. Allen Species and Evolution (Island, Washington, 2004).

4. Simmons, A. D.\& Thomas C.D. Am Nat. 164, 378-395 (2004)

5. Travis, I.M.L \&Dytham, C.Evol. Ecal. Res. 4, $119-1129$ (2002).

6. Lever, C. The Cane Taad The Histary and Ecalogy of a Sercessful Calonist Westbury, Otley, West Yorkshire, 2001).

7. Smith, M. A. \& Green, D. M. Ecography 28, 110-128 (2005)

8. Choi, L,Shim, 1.H.\& Ricklets, R.E.J.Exp Zoal 299A, 99-102 (2008)

9. Freeland W.I \&.Martin, K.C. Aust. Wildl Res 12,555-599 (1985)

10. Thomas, C D.et al Nature 411, 577-581(2001).

Sup plementary information accompanies this commun ication on Nature's website.

Recelved 24 November 2005; accepted 24 January 2006.

Competing fin ancial interests; declared none.

doi:10.1038/439803a 